J. Dairy Sci. 97:5533-5542

http://dx.doi.org/10.3168/jds.2014-8129

(C) American Dairy Science Association ${ }^{\circledR}, 2014$.

\title{
Effect of merging levels of locomotion scores for dairy cows on intra- and interrater reliability and agreement
}

\author{
Andrés Schlageter-Tello, ${ }^{* 1}$ Eddie A. M. Bokkers, $†$ Peter W. G. Groot Koerkamp,‡ Tom Van Hertem,§ \\ Stefano Viazzi,§ Carlos E. B. Romanini,§ Ilan Halachmi,\# Claudia Bahr,§ Daniël Berckmans,§ \\ and Kees Lokhorst* \\ *Wageningen UR Livestock Research, PO Box 65, 8200 AB Lelystad, the Netherlands \\ †Animal Production Systems Group, Wageningen University, PO Box 338, $6700 \mathrm{AH}$, Wageningen, the Netherlands \\ †Farm Technology Group, Wageningen University, PO Box 317, $6700 \mathrm{AH}$, Wageningen, the Netherlands \\ §Division Measure, Model \& Manage Bioresponses, KU Leuven, PO Box 2456, 3001 Heverlee, Belgium \\ \#Institute of Agricultural Engineering-Agricultural Research Organization, PO Box 6, Bet-Dagan 50250, Israel
}

\begin{abstract}
Locomotion scores are used for lameness detection in dairy cows. In research, locomotion scores with 5 levels are used most often. Analysis of scores, however, is done after transformation of the original 5-level scale into a 4-, 3-, or 2-level scale to improve reliability and agreement. The objective of this study was to evaluate different ways of merging levels to optimize resolution, reliability, and agreement of locomotion scores for dairy cows. Locomotion scoring was done by using a 5-level scale and 10 experienced raters in 2 different scoring sessions from videos from 58 cows. Intra- and interrater reliability and agreement were calculated as weighted kappa coefficient $(\mathrm{kW})$ and percentage of agreement (PA), respectively. Overall intra- and interrater reliability and agreement and specific intra- and interrater agreement were determined for the 5-level scale and after transformation into 4-, 3-, and 2-level scales by merging different combinations of adjacent levels. Intrarater reliability $(\mathrm{kw})$ ranged from 0.63 to 0.86 , whereas intrarater agreement (PA) ranged from 60.3 to $82.8 \%$ for the 5 -level scale. Interrater $\mathrm{kw}=0.28$ to 0.84 and interrater $\mathrm{PA}=22.6$ to $81.8 \%$ for the 5 -level scale. The specific intrarater agreement was $76.4 \%$ for locomotion level 1, $68.5 \%$ for level $2,65 \%$ for level $3,77.2 \%$ for level 4 , and $80 \%$ for level 5 . Specific interrater agreement was $64.7 \%$ for locomotion level 1, $57.5 \%$ for level 2, $50.8 \%$ for level $3,60 \%$ for level 4 , and $45.2 \%$ for level 5 . Specific intra- and interrater agreement suggested that levels 2 and 3 were more difficult to score consistently compared with other levels in the 5-level scale. The acceptance threshold for overall intra- and interrater reliability ( $\kappa \mathrm{w}$ and $\kappa \geq 0.6)$ and agreement $(\mathrm{PA} \geq 75 \%)$ and specific intra- and interrater agreement $(\geq 75 \%$ for
\end{abstract}

Received March 12, 2014.

Accepted June 6, 2014.

${ }^{1}$ Corresponding author: andres.schlagetertello@wur.nl all levels within locomotion score) was exceeded only for the 2-level scale when the 5 levels were merged as (12)(345) or (123)(45). In conclusion, when locomotion scoring is performed by experienced raters without further training together, the lowest specific intra- and interrater agreement was obtained in levels 2 and 3 of the 5-level scale. Acceptance thresholds for overall intra- and interrater reliability and agreement and specific intra- and interrater agreement were exceeded only in the 2-level scale.

Key words: cattle, lameness, observer, resolution

\section{INTRODUCTION}

Locomotion scoring is a procedure used to indicate the quality of locomotion of cows. Raters assess gait and posture traits of cows and assign a locomotion score according to their judgment. Locomotion scores are often used to detect lameness in dairy cows (Whay, 2002; Flower and Weary, 2009). A cow is classified as lame when a predefined threshold on the scale is exceeded (Sprecher et al., 1997; Winckler and Willen, 2001; Chapinal et al., 2009).

Locomotion scores are sensitive to variation for intra- and interrater comparisons (Engel et al., 2003; O'Callaghan et al., 2003; Thomsen et al., 2008). Following Kottner et al. (2011), reliability is defined as the capability of raters to differentiate between levels within the score (e.g., lame and nonlame), whereas agreement indicates the capability of raters to assign identical scores to the same cow. Reliability and agreement are important indicators of consistency and reproducibility of measurements (Martin and Bateson, 1993; Kottner et al., 2011). It is also stated that measurements with low reliability and agreement cannot be valid (Franzen, 2000). Reliability and agreement can be calculated by comparing data scores assigned to a cow by the same rater under similar conditions at different times (intra- 
rater reliability and agreement) or by comparing scores from 2 or more raters assigned to the same cow under similar conditions (interrater reliability and agreement; Martin and Bateson, 1993).

From a practical standpoint, high reliability and agreement for locomotion scores are important for generating consistent and comparable data for lameness control programs (DairyCo, 2007; Welfare Quality, 2009). In addition, locomotion scores are used as reference for calibration and validation in the development of different types of automatic locomotion scoring systems (Chapinal et al., 2010; de Mol et al., 2013; Viazzi et al., 2013).

Resolution is defined as the smallest change in locomotion that can be detected by the locomotion score and it is expressed in the number of levels of the scale (Martin and Bateson, 1993). A locomotion score with a multiple-level scale (and high resolution) is desirable because it would allow a better description of locomotion quality. In addition, a multiple-level locomotion score would allow users to take different actions with cows scored in different levels, as suggested for some locomotion scores (DairyCo, 2007). A large number of levels in a scale would provide more freedom to researchers and decision makers for data handling.

It is common practice to decrease the number of levels within a scale by merging adjacent levels to improve reliability or agreement (e.g., percentage of agreement). From a practical point of view, locomotion scores are also merged to create a binary classification of cows as lame or nonlame (Winckler and Willen, 2001; Channon et al., 2009; Main et al., 2010). However, no standard method yet exists for merging levels. Therefore, the decision as to which levels should be merged depends mainly on the criteria of the user of the locomotion score. When merging levels, resolution is lost from the locomotion score, a loss that tends to increase as fewer levels are used in the scale (Engel et al., 2003). To optimize reliability, agreement, and resolution of locomotion scores when levels are merged, it is important to understand the agreement in specific levels within the scale of a locomotion score. Thus, by knowing agreement of raters at each specific level, the level at which raters perform worst could be identified and merged.

To increase the practical value of locomotion scores and to support further development of automatic lameness detection systems, insight is needed in the reliability, agreement, and resolution of locomotion scores for dairy cows. Therefore, the objective of this study was to evaluate different ways of merging levels to optimize resolution, reliability, and agreement of locomotion scores for dairy cows.

\section{MATERIALS AND METHODS}

\section{Video Recording}

Video recording was performed at a dairy farm with 1,100 milking cows located in Israel and previously described by Van Hertem et al. (2013). Cows walking through an alley (1.5 m wide, $7 \mathrm{~m}$ long) on a concrete floor were recorded with a NikonD7000 camera (Nikon Corp., Tokyo, Japan) equipped with a Nikkor DX AF-S 18-105 mm G ED lens (Nikon Corp.). The walking alley was situated at the exit of the milking area. To obtain flank views of cows, the camera was positioned $4 \mathrm{~m}$ perpendicular to the progression line of the alley and $1.35 \mathrm{~m}$ above ground level. Video records (.mov file format) had a resolution of $1,920 \times 1,080$ pixels at a frame rate of 25 frames per secondCamera settings were as follows: focal length $=18 \mathrm{~mm}$, shutter speed $=1 / 40$, aperture value $=3.5$, and ISO speed: 5000 . Because the video recordings were performed at night, external light sources were used to allow a clear observation of cows. To obtain individual video records of each cow, the video records were edited with Quick Time 7 Pro (Apple Inc., Cupertino, CA).

\section{Locomotion Score}

Locomotion scoring was performed using a 5-level scale that was based on judging 5 gait and posture traits: asymmetric gait, arched back, reluctance to bear weight, tracking up, and head bob, as described by Flower and Weary (2006). In short, cows scored in level 1 had a smooth and fluid movement; cows in level 2 had an imperfect locomotion but were able to move freely; cows in level 3 had a compromised ability to move freely; for cows in level 4, the ability to move freely was obviously diminished; and for cows in level 5 , the ability to move was severely restricted.

\section{Video Selection}

Video records of all individual cows in the herd were stored in a video data set. Each video record was scored for locomotion according the previously described 5-level scale by 1 experienced rater [intrarater reliability/agreement: weighted kappa $(\mathbf{k w})=0.86 /$ percentage of agreement $=84.5 \%$ ] who did not participate in the experiment. Video records for each level within the 5 -level scale were selected randomly from the video data set. A video record was included in the experiment only if the cow made at least 4 steps without stopping and sufficient contrast existed between the cow and the background. If a video record did not meet the quality 
criteria, a new video record was selected randomly from the video data set until a predetermined number of 12 video records per level was reached. For level 5, only 8 video records were available that met the criteria. Therefore, 2 extra video records were added for level 3 because this level appeared to be the most difficult to assess consistently. The 58 video records selected were from 58 different cows. The number of video records used in the present experiment was determined using reporting reliability and agreement for locomotion scoring in dairy cows from the literature as reference (Flower and Weary, 2006; Thomsen et al., 2008; Channon et al., 2009) and to avoid fatigue of raters for scoring a large number of video records. In addition, because the similar number of videos in each level of the scale was an important part of the experimental design, the lack of video records classified as level 5 limited the total number of videos that could be included in the experiment.

\section{Raters and Scoring Sessions}

Locomotion scoring was performed by 10 experienced raters with different backgrounds and originally trained using different locomotion scores (Table 1). Raters were not informed about the objectives of the study, the number of different video records used, or the randomizations performed during the experiment.

The 58 video records were shown to the 10 raters in 2 scoring sessions separated by at least $4 \mathrm{~d}$. Each scoring session was split in 6 parts, in which raters scored the 58 video records each time again for either locomotion score or 1 of the 5 gait and posture traits independently. Each part lasted approximately $30 \mathrm{~min}$, including $10 \mathrm{~min}$ for instruction and $20 \mathrm{~min}$ for scoring.

In both sessions, the raters received a short instruction on locomotion scoring or scoring one of the gait and posture traits at the start of each part. The instruction consisted of showing 2 video records per level of the 5-level scale. The instruction was done by the experienced rater, who was also responsible for the selection of the video records. Video records used for instruction were not included in the experiment. The instruction was the only time during which raters were allowed to discuss scoring.

For instruction and scoring, video records were shown with a projector on a white screen. Every video record was shown twice. The scoring was performed using an online interface that stored scores from raters directly in a database. The order in which locomotion and gait and posture traits were shown was randomly chosen in every session. In addition, to avoid cow recognition, video records were shown in a different random order in each part. All randomizations were done using an online random number generator (www.random.org). For practical reasons, it was not possible to have all raters in the same room at the same time; therefore, the experiment was conducted with 4 groups (Table 1 ).

\section{Statistical Analysis}

In the present study, only data from locomotion scores were analyzed and presented. Data related to scoring of individual gait and posture traits will be presented in another article. Intra- and interrater reliability and agreement were calculated for the original 5-level scale and after merging different combinations of adjacent levels to create 4-, 3-, and 2-level scales. Intrarater reliability and agreement were calculated by comparing the scores from the same cow in 2 different sessions. For both sessions, interrater reliability and agreement were calculated by comparing the scores of the same cow assigned to 2 different raters.

Intra- and interrater reliability was calculated as Kw (Cohen, 1968) for the 5-, 4-, and 3-level scales; the kappa coefficient $(\boldsymbol{\kappa})$ was calculated for the 2-level scale (Cohen, 1960). Intra- and interrater agreement was expressed as exact percentage of agreement (PA)

Table 1. Background and experience of 10 raters participating in the study

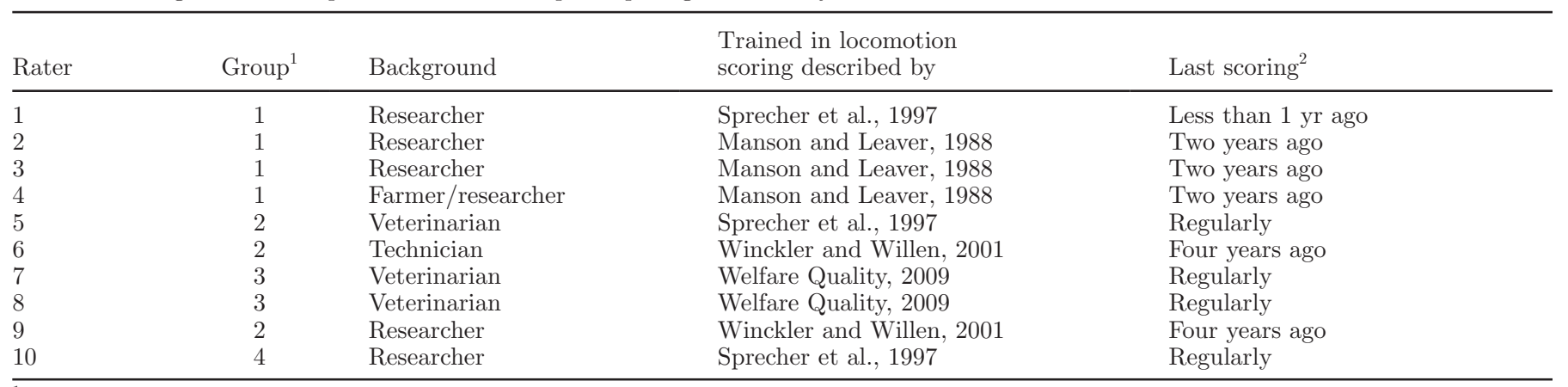

${ }^{1}$ Same number indicates that raters conducted scoring sessions together.

${ }^{2}$ Indicates how long ago the raters regularly performed locomotion scoring in relation to the start of the experiment. 
Table 2. Distribution of locomotion scores assigned using a 5-level scale by 10 raters

\begin{tabular}{|c|c|c|c|c|c|c|c|c|c|c|c|}
\hline Score & \multicolumn{11}{|c|}{ Rater $^{1}$} \\
\hline Level 2 & 12 & 15 & 22 & 17 & 16 & 16 & 18 & 21 & 24 & 20 & 13 \\
\hline Level 3 & 14 & 14 & 13 & 11 & 18 & 8 & 16 & 16 & 14 & 18 & 17 \\
\hline Level 4 & 12 & 13 & 13 & 13 & 15 & 11 & 12 & 9 & 6 & 12 & 12 \\
\hline Level 5 & 8 & 3 & 2 & 0 & 1 & 9 & 3 & 2 & 1 & 2 & 6 \\
\hline
\end{tabular}

${ }^{1}$ Values are averages from sessions 1 and 2 .

${ }^{2}$ Distribution of locomotion scores according the experienced rater selecting video records.

for 5-, 4-, 3-, and 2-level scales; 95\% CI were calculated for $\kappa \mathrm{w}$ and $\kappa$ (Fleiss et al., 1969) and Clopper-Pearson CI (Brown et al., 2001) were calculated for PA. When expressed as $\mathrm{\kappa W}$ and $\kappa$, reliability can be classified as follows: poor ( $\kappa \mathrm{W}$ and $\kappa<0.00$ ), slight ( $\kappa \mathrm{W}$ and $\kappa=$ $0.00-0.19$ ), fair ( $\kappa \mathrm{w}$ and $\kappa=0.20-0.39$ ), moderate ( $\mathrm{\kappa w}$ and $\kappa=0.4-0.59$ ), substantial ( $\kappa \mathrm{w}$ and $\kappa=0.6-0.79$ ), or excellent ( $\kappa \mathrm{w}$ and $\kappa=0.8-1$ ) (Landis and Koch, 1977). The commonly accepted threshold for good reliability is indicated at $\kappa \mathrm{w}$ and $\kappa \geq 0.6$ (Gibbons et al., 2012). The commonly accepted threshold for agreement is $\geq 75 \%$ (Burn and Weir, 2011).

Overall intrarater reliability and agreement were calculated by creating a cross table that included all comparisons for the same rater. Overall interrater reliability and agreement were calculated with a cross table including all pairwise comparisons for raters and sessions.

Cross tables used to calculate overall intra- and interrater reliability and agreement were used to calculate the percentage of specific agreement. Percentage of specific agreement is based on the concept of positive and negative agreement (Cicchetti and Feinstein, 1990). Specific agreement indicates the capability of raters to agree on a specific level of the scale. The specific intrarater agreement indicates the average in which a single rater agrees in scoring a cow in the same level in 2 sessions. Specific interrater agreement indicates the average in which 2 raters agree in scoring a cow in the same level in 2 sessions. The confidence limits for the specific agreement were calculated with the delta method as proposed by Graham and Bull (1998). No established acceptance threshold exists for specific intra- and interrater agreement. Therefore, the same acceptance threshold as for inter- and intrarater agreement (PA $\geq 75 \%)$ was used.

\section{RESULTS}

\section{Distribution of Locomotion Scores}

The distribution of scores for 10 raters using the 5 -level scale is shown in Table 2. All raters scored all
58 video records. However, because of practical issues, some data were missed. Thus, rater 1 scored 56 video records in session 2 , rater 4 scored 56 video records in session 1 and 53 in session 2, and rater 6 scored 57 video records in session 1 . We observed large variation between raters in the distribution of scores. Three to 18 video records were scored as level 1; between 13 and 24 were scored as level 2; between 8 and 18 were scored as level 3; between 6 and 15 were scored as level 4; and between zero and 9 were scored as level 5 (Table 2).

\section{Intra- and Interrater Reliability and Agreement for Five-Level Scale}

Intra- and interrater reliability and agreement are shown in Table 3. Intrarater reliability ranged from 0.63 to 0.86 ; therefore, all raters exceeded the acceptance threshold for $\mathrm{kw}$. Intrarater agreement ranged from 60.3 to $82.8 \%$; the acceptance threshold for intrarater agreement was exceeded for raters $3,8,9$, and 10 .

Interrater reliability ranged from 0.51 to 0.84 in session 1 and from 0.28 to 0.82 in session 2. The acceptance threshold for interrater reliability was exceeded in 39 of 45 pairwise comparisons in session 1 and in 29 of 45 pairwise comparisons in session 2. Interrater agreement ranged from 43.1 to $81.8 \%$ in session 1 and from 22.6 to $75.8 \%$ in session 2 . The acceptance threshold for interrater agreement was exceeded in 3 of 45 pairwise comparisons in session 1 and in 1 of 45 pairwise comparisons in session 2. Some pairwise comparisons exceeded the acceptance threshold for $\mathrm{\kappa w}$, even with $\mathrm{PA}$ values below $50 \%$ (e.g., comparison rater 4 and rater 5 in session 1 ).

Although each video record in the experiment was shown 12 times in each session (24 times in total), raters indicated no cow memorization when asked at the end of session 2 .

\section{Overall Intra- and Interrater Reliability and Agreement}

Overall intrarater reliability and agreement for different 5-, 4-, 3-, and 2-level scales are shown in Table 
Table 3. Intrarater reliability and agreement (in the diagonal) and interrater reliability and agreement for session 1 (above the diagonal) and session 2 (under the diagonal) for pairwise comparison of 10 raters for a locomotion score with a 5-level scale

\begin{tabular}{|c|c|c|c|c|c|c|c|c|c|c|c|}
\hline \multirow[b]{2}{*}{ Session 2 rater } & \multirow[b]{2}{*}{ Parameter $^{1}$} & \multicolumn{10}{|c|}{ Session 1 rater } \\
\hline & & 1 & 2 & 3 & 4 & 5 & 6 & 7 & 8 & 9 & 10 \\
\hline & PA & 66.1 & 68.9 & 63.7 & 67.9 & 58.6 & 77.2 & 72.4 & 58.6 & 68.9 & 60.3 \\
\hline \multirow[t]{2}{*}{2} & $\kappa \mathrm{W}$ & 0.72 & 0.77 & 0.73 & 0.72 & 0.64 & 0.78 & 0.69 & 0.62 & 0.71 & 0.61 \\
\hline & PA & 66.1 & 72.4 & 67.2 & 69.6 & 50.0 & 75.4 & 65.1 & 55.2 & 67.2 & 50.0 \\
\hline 3 & $\kappa w$ & 0.70 & 0.71 & 0.82 & 0.70 & 0.60 & 0.72 & 0.75 & 0.75 & 0.60 & 0.60 \\
\hline & PA & 43.1 & 37.7 & 32.1 & 64.7 & 46.4 & 81.8 & 71.4 & 59.0 & 71.4 & 57.2 \\
\hline \multirow[t]{2}{*}{5} & $\kappa \mathrm{W}$ & 0.69 & 0.70 & 0.75 & 0.42 & 0.78 & 0.63 & 0.57 & 0.61 & 0.58 & 0.72 \\
\hline & PA & 57.1 & 56.9 & 63.8 & 28.3 & 67.2 & 47.4 & 43.1 & 50.0 & 44.8 & 60.3 \\
\hline \multirow[t]{2}{*}{6} & kw & 0.67 & 0.71 & 0.57 & 0.57 & 0.66 & 0.70 & 0.75 & 0.70 & 0.79 & 0.70 \\
\hline & PA & 60.7 & 63.8 & 41.4 & 54.7 & 50.0 & 63.2 & 71.9 & 64.9 & 75.4 & 61.4 \\
\hline \multirow[t]{2}{*}{7} & $\kappa \mathrm{W}$ & 0.60 & 0.57 & 0.65 & 0.39 & 0.68 & 0.62 & 0.66 & 0.66 & 0.65 & 0.58 \\
\hline & PA & 51.8 & 48.3 & 58.6 & 39.6 & 62.1 & 51.7 & 60.3 & 62.1 & 62.1 & 48.3 \\
\hline 10 & PA & 53.6 & 58.6 & 48.3 & 50.9 & 62.1 & 75.8 & 44.8 & 41.4 & 60.4 & 81.0 \\
\hline
\end{tabular}

${ }^{1}$ Reliability was expressed as weighted kappa ( $\left.\mathrm{kw}\right)$ and agreement as percentage of agreement (PA).

4. Overall intrarater reliability exceeded the acceptance threshold for the 5-level scale and all different combinations for 4-, 3-, and 2-level scales. The overall intrarater agreement acceptance threshold was exceeded in most of the 4-level scales, except for the combination 123(45), and all 3- and 2-level scales (Table 4). The CI for intrarater reliability showed no differences for most of the 5-, 4-, 3-, and 2-level scales (Table 4). Overall intrarater agreement tended to increase (by approximately 7 percentage points) every time the scale decreased by 1 level.
Overall interrater reliability and agreement for 5-, 4-, $3-$, and 2-level scales are shown in Table 4 . The overall interrater reliability acceptance threshold was exceeded for the 5-level scale and most of the combinations for 4-, 3, and 2-level scales (Table 4). The interrater agreement acceptance threshold was exceeded for most of the 3- and 2-level scales (Table 4). The CI for interrater reliability indicated no differences for 5-level locomotion score and the different 4-, 3-, and 2-level scales, with 2 exceptions: for 3-level scale combination $1(234) 5$ and 2-level scale combination (1234)5 (Table 4). Over-

Table 4. Overall intra- and interrater reliability and agreement for the original 5-level scale and for transformation into 4-, 3-, and 2-level scales ${ }^{1}$

\begin{tabular}{|c|c|c|c|c|c|}
\hline Scale & Combination $^{2}$ & \multicolumn{2}{|c|}{ Intrarater } & \multicolumn{2}{|c|}{ Interrater } \\
\hline 5-level & - & $0.77(0.74-0.80)$ & $71.4(67.7-75.1)$ & $0.65(0.64-0.66)$ & $57.1(55.7-58.4)$ \\
\hline \multirow{3}{*}{ 4-level } & $1(23) 45$ & $0.77(0.73-0.81)$ & $82.1(78.9-85.2)$ & $0.61(0.60-0.63)$ & $70.6(69.3-71.8)$ \\
\hline & $12(34) 5$ & $0.75(0.72-0.80)$ & $78.1(74.7-81.5)$ & $0.63(0.61-0.64)$ & $67.4(66.1-68.7)$ \\
\hline & $123(45)$ & $0.77(0.75-0.80)$ & $73.3(69.7-76.9)$ & $0.67(0.65-0.68)$ & $62.1(60.7-63.4)$ \\
\hline \multirow{4}{*}{ 3-level } & $(12)(34) 5$ & $0.77(0.73-0.83)$ & $86.8(84.1-89.6)$ & $0.66(0.64-0.68)$ & $80.0(79.0-81.1)$ \\
\hline & $12(345)$ & $0.75(0.71-0.79)$ & $80.0(76.7-83.3)$ & $0.65(0.64-0.67)$ & $72.6(71.4-73.8)$ \\
\hline & $1(234) 5$ & $0.73(0.67-0.79)$ & $89.3(86.7-91.8)$ & $0.53(0.51-0.56)$ & $81.7(80.7-82.8)$ \\
\hline & (123)45 & $0.80(0.75-0.85)$ & $90.9(88.5-93.2)$ & $0.64(0.62-0.66)$ & $83.6(82.6-84.6)$ \\
\hline \multirow[t]{3}{*}{ 2-level } & $1(2345)$ & $0.71(0.64-0.79)$ & $91.2(88.9-93.5)$ & $0.57(0.54-0.60)$ & $86.9(86.0-87.8)$ \\
\hline & $(12)(345)$ & $0.78(0.72-0.83)$ & $88.7(86.1-91.2)$ & $0.70(0.68-0.72)$ & $85.2(84.2-86.2)$ \\
\hline & $(123)(45)$ & $0.81(0.75-0.86)$ & $92.8(90.7-94.9)$ & $0.70(0.67-0.72)$ & $88.6(87.7-89.5)$ \\
\hline
\end{tabular}

${ }^{1}$ Reliability was expressed as weighted kappa $(\kappa \mathrm{w})$ or kappa ( $\kappa$, for 2-level scale) coefficients, and agreement was expressed as percentage of agreement (PA) and 95\% CI.

${ }^{2}$ Parentheses indicate levels merged from the original 5-level scale. 
Table 5. Specific intrarater agreement for individual levels and 95\% CI of a 5-level scale and the individual levels of 4-, 3-, and 2-level scales

\begin{tabular}{llcccc}
\hline Scale & Combination $^{1}$ & Level 1 $(\mathrm{CI})$ & Level 2 $(\mathrm{CI})$ & Level 3 $(\mathrm{CI})$ & Level 4 (CI) \\
\hline 5-level & - & $76.4(70.1-82.7)$ & $68.5(63.0-74.0)$ & $65.0(58.6-71.5)$ & $77.2(71.2-83.2)$ \\
4-level & $(12) 345$ & $88.8(86.1-91.6)$ & $65.0(58.6-71.5)$ & $77.2(71.2-83.2)$ & $80.0(68.4-91.6)$ \\
& $1(23) 45$ & $76.4(70.1-82.7)$ & $85.9(83.0-88.8)$ & $77.2(71.2-83.2)$ & $80.0(68.4-91.6)$ \\
& $12(34) 5$ & $76.4(70.1-82.7)$ & $68.5(63.0-74.0)$ & $85.3(82.0-88.6)$ & $80.0(68.4-91.6)$ \\
& $123(45)$ & $76.4(70.1-82.7)$ & $68.5(63.0-74.0)$ & $65.0(58.6-71.5)$ & $85.5(81.1-89.9)$ \\
3-level & $(12) 3(45)$ & $88.8(86.1-91.6)$ & $65.0(58.6-71.5)$ & $85.5(81.1-89.9)$ & \\
& $1(23)(45)$ & $76.4(70.1-82.7)$ & $85.9(83.0-88.8)$ & $85.5(81.1-89.9)$ & \\
& $(12)(34) 5$ & $88.8(86.1-91.6)$ & $85.3(82.0-88.6)$ & $80.0(68.4-91.6)$ & \\
& $12(345)$ & $76.4(70.1-82.7)$ & $68.5(63.0-74.0)$ & $88.6(85.9-91.4)$ & \\
& $1(234) 5$ & $76.4(70.1-82.7)$ & $93.0(91.3-94.8)$ & $80.0(68.4-91.6)$ & \\
2-level & $(123) 45$ & $95.2(93.7-96.7)$ & $77.2(71.2-83.2)$ & $80.0(68.4-91.6)$ & \\
& $1(2345)$ & $76.4(70.1-82.7)$ & $94.6(93.1-96.1)$ & & \\
& $(12)(345)$ & $88.8(86.1-91.6)$ & $88.6(85.9-91.4)$ & & \\
& $(123)(45)$ & $95.2(93.7-96.7)$ & $85.5(81.2-89.9)$ & & \\
\hline
\end{tabular}

${ }^{1}$ Parentheses indicate levels merged from the original 5 -level scale.

all interrater agreement increased (by approximately 10 percentage points) every time the scale of the locomotion score decreased by 1 level.

\section{Specific Intra- and Interrater Agreement}

The specific intra- and interrater agreement for the 5-level scale and the specific levels for different 4-, 3-, and 2-level scales are shown in Tables 5 and 6 . The specific intrarater agreement for the original 5 -level scale were $76 \%$ for level 1, $69 \%$ for level $2,65 \%$ for level 3 , $77 \%$ for level 4 , and $80 \%$ for level 5 (Table 5 ). The CI indicated that level 3 presented lower specific intrarater agreement than level 4. Scales exceeding the acceptance threshold for specific intrarater agreement in all lev- els were a 4-level scale combination [1(23)45]; 3-level scale combinations $[1(23)(45),(12)(34) 5,1(234) 5$, and (123)45]; and all 2-level scales.

The specific interrater agreement for the 5-level scale and different 4-, 3-, and 2-level scales are shown in Table 6 . The specific interrater agreement for 5-level scales were $64.7,57.5,50.8,60.0$, and $45.2 \%$ for levels 1 , $2,3,4$, and 5 , respectively (Table 6 ). The CI indicated that specific interrater agreement was lower for levels 3 and 5 than for levels 1,2 , and 4 . Scales exceeding the acceptance threshold for specific intrarater agreement in all levels were the 2-level scale combinations (12) (345) and (123)(45).

Specific interrater agreement had similar values for level 1 (session $1=63.4 \%$, session $2=65.8 \%$ ) and

Table 6. Specific interrater agreement for individual levels and 95\% CI of a 5-level scale and the individual levels of 4-, 3-, and 2-level scales

\begin{tabular}{llcccc}
\hline Scale & Combination $^{1}$ & Level 1 $(\mathrm{CI})$ & Level 2 $(\mathrm{CI})$ & Level 3 $(\mathrm{CI})$ & Level $4(\mathrm{CI})$ \\
\hline 5-level & - & $64.7(62.2-67.2)$ & $57.5(55.4-59.5)$ & $50.8(48.5-53.2)$ & $60.0(57.3-62.5)$ \\
4-level & $(12) 345$ & $85.2(84.2-86.3)$ & $50.8(48.5-53.2)$ & $60.0(57.5-62.5)$ & $45.2(39.6-50.7)$ \\
& $1(23) 45$ & $64.7(62.2-67.2)$ & $78.4(77.2-79.6)$ & $60.0(57.3-62.5)$ & $45.2(39.6-50.7)$ \\
& $12(34) 5$ & $64.7(62.2-67.2)$ & $57.5(55.4-59.5)$ & $77.8(76.5-79.1)$ & $45.2(39.6-50.7)$ \\
& $123(45)$ & $64.7(62.2-67.2)$ & $57.5(55.4-59.5)$ & $50.8(48.5-53.2)$ & $77.1(75.3-78.9)$ \\
3 -level & $(12) 3(45)$ & $85.2(84.2-86.3)$ & $50.8(48.5-53.2)$ & $77.1(75.3-78.9)$ & \\
& $1(23)(45)$ & $64.7(62.2-67.2)$ & $78.4(77.2-79.6)$ & $77.1(75.3-78.9)$ & \\
& $(12)(34) 5$ & $85.2(84.2-86.3)$ & $77.8(76.5-79.1)$ & $45.2(39.6-50.7)$ & \\
& $12(345)$ & $64.7(62.2-67.2)$ & $57.5(55.4-59.5)$ & $85.1(84.1-86.2)$ & \\
& $1(234) 5$ & $64.7(62.2-67.2)$ & $88.1(87.3-88.8)$ & $45.2(39.6-50.7)$ & \\
& $(123) 45$ & $92.4(91.8-93.0)$ & $60.0(57.3-62.5)$ & $45.2(39.6-50.7)$ & \\
2-level & $1(2345)$ & $64.7(62.2-67.2)$ & $91.9(91.4-92.6)$ & & \\
& $(12)(345)$ & $85.2(84.2-86.3)$ & $85.1(84.1-86.2)$ & & \\
& $(123)(45)$ & $92.4(91.8-93.0)$ & $77.1(75.3-78.9)$ & & \\
& $(1234) 5$ & $97.3(96.9-97.6)$ & $45.2(39.6-50.7)$ & & \\
\end{tabular}

\footnotetext{
${ }^{1}$ Parentheses indicate levels merged from the original 5 -level scale.
} 
level 4 (session $1=60.8 \%$, session $2=59.2 \%$ ). The CI suggest that specific interrater agreements for level 2 in session $1(64.3 \%)$ and session $2(49.8 \%)$ were different (Figure 1). The CI for specific interrater agreements for level 3 of session 1 (56.5\%) and session 2 (45.1\%) were different. Although specific interrater agreement for level 5 showed large variation for session 1 (41.9\%) and session 2 (49.1\%), the CI suggest no differences between sessions (Figure 1).

\section{DISCUSSION}

In literature, reliability or agreement are usually reported briefly to indicate the level of training of the raters assessing locomotion scores (Rutherford et al., 2009; Barker et al., 2010; Ito et al., 2010). However, important information about the experimental methodology for correct interpretation of reliability and agreement estimates is commonly omitted; for example, the total number of cows and the number of cows assigned to each level of the scale, the communication allowed among raters, or randomizations performed during the experiment (Danscher et al., 2009; Katsoulos and Christodoulopoulos, 2009; Main et al., 2010). Reliability or agreement is usually estimated using the total number of animals on one or more farms, where the total number of cows in levels 1 and 2 is greater than the number of cows in levels 3, 4, and 5 (Winckler and Willen, 2001; Thomsen et al., 2008; Channon et al., 2009). Reliability strongly depends on the distribution in the population sample (de Vet et al., 2006). Thus, when the total number of animals within the farm is used, reliability estimates may present values under the acceptance threshold, not due to an effect of rater but by the effect of the sample distribution (Hoehler, 2000; de Vet et al., 2006). A solution for this problem is to estimate reliability and agreement using similar numbers of individuals in each level of the scale or to report distribution of the population sample for a better interpretation of reliability estimates (Burn and Weir, 2011). In this regard, the methodology described herein may be used as a guideline for future studies using locomotion scoring or other indicators measured with visual scores. Further details for methodologies for reporting agreement and reliability were described by Kottner et al. (2011).

Different raters showed large variation in scoring the same cow for locomotion on a 5-level scale. In this regard, different backgrounds and initial training of raters might be factors explaining the variation in scores. The 10 raters exceeded the acceptance threshold for intrarater reliability. This indicates that disagreements were mainly due to 1 level, whereas disagreements for 2 or 3 levels are less common (Winckler and Willen, 2001;

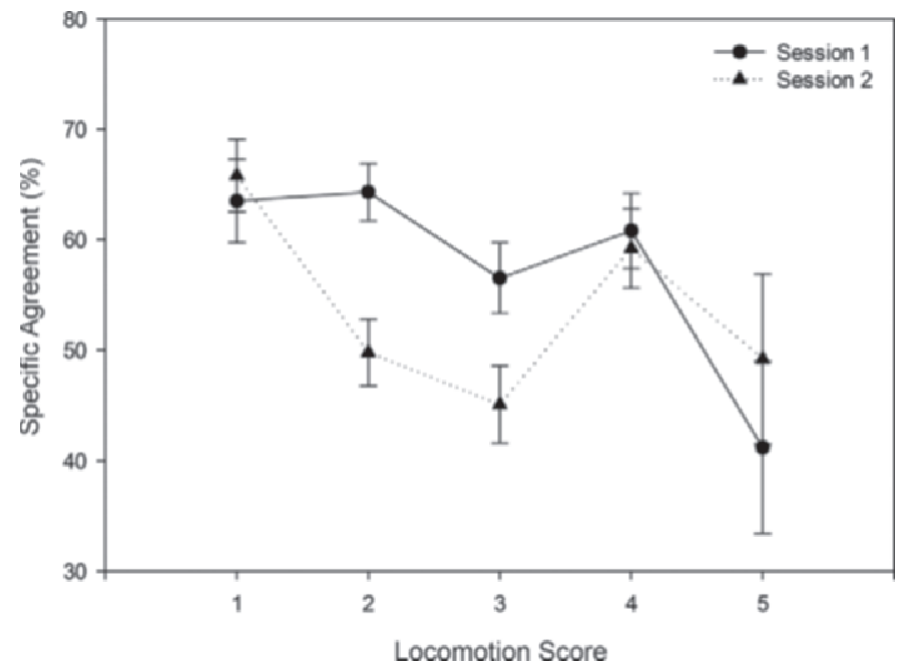

Figure 1. Specific interrater agreement in 2 sessions for a locomotion score with a 5 -level scale (bars indicate 95\% CI).

O'Callaghan et al., 2003; Channon et al., 2009). Therefore, raters were able to differentiate properly between levels of the 5-level ordinal scale. Intrarater agreement was, in most cases, below the acceptance threshold, indicating that it is difficult even for experienced raters to obtain exact agreement in a 5-level scale. Values for intrarater reliability and agreement suggest that raters in the current experiment were experienced. Large ranges in values for interrater reliability and agreement indicate that, although raters were experienced, they did not have further training together, which is thought to be an important factor in interrater reliability and agreement (Kazdin, 1977; March et al., 2007).

Intrarater reliability values in the present experiment were higher than values reported for a similar locomotion score with a 5-level scale performed by experienced raters after a short training, with $\mathrm{kw}$ ranging from 0.38 to 0.64 (Thomsen et al., 2008). Intrarater agreement values in the present study were higher than values reported for a similar 5-level scale, where PA $=56 \%$ (O'Callaghan et al., 2003). Differences from other studies for intrarater reliability and agreement may be explained by the different levels of experience of the raters participating in different experiments (March et al., 2007; Gibbons et al., 2012) and the practical conditions in which the locomotion scoring was performed: scoring from video in the present study versus live scoring in other studies (O'Callaghan et al., 2003; Thomsen et al., 2008). Results obtained in the present experiment were similar to others reported in the literature for interrater reliability (Thomsen et al., 2008; Hoffman et al., 2013) and interrater agreement (Winckler and Willen, 2001; Katsoulos and Christodoulopoulos, 2009) for similar 5-level locomotion scores. 
Merging levels had no effect on the overall intra- and interrater reliability for most combinations, with some exceptions for the 3-level scale combination 1(234)5 and 2-level scale combinations 1(2345) and (1234)5, which presented $\kappa \mathrm{w}$ and $\kappa$ estimates lower than the acceptance threshold. This may be explained because merging 3 or 4 levels within the 5-level scale affected the distribution of the population sample, which also affected the reliability. Other authors reported an increment in interrater reliability estimates when expressed as $\kappa$ coefficient when levels were merged from a 5 - to a 2-level scale (March et al., 2007; Channon et al., 2009). However, $\kappa$ coefficient is an inappropriate statistic to estimate reliability in ordinal scales (Kottner et al., 2011). Increment in interrater agreement has been reported previously when a locomotion score with 4-level scale was merged into a 2-level scale (Rutherford et al., 2009; Barker et al., 2010).

Relatively low values for specific intra- and interrater agreement in levels 2 and 3 for the original 5-level scale suggest that scoring of these 2 levels is difficult for experienced raters. This means that cows with slight locomotion alterations (or early stage lameness) are difficult to identify, even by experienced raters. Winckler and Willen (2001) reported that the greatest variation (in a similar 5-level scale as that used in the present study) was between levels 1 and 2, which were also the levels in which most of the cows were scored, whereas in the present study, similar numbers of cows were present in all 5 levels of the scale. More uncertain is the explanation for the low specific interrater agreement in level 5 , which might be due to the smaller number of video records in this level of the scale. However, the specific intrarater agreement for level $5(80.0 \%)$ was almost twice as high as the specific interrater agreement for level 5 (45.2\%), which indicates a disagreement between raters scoring level 5 of the scale, probably due to the lack of training of raters together. Low specific interrater agreement in level 5, however, has minor practical implications because the prevalence of cows scored as level 5 is commonly low in farms because cows are treated or culled before cows reach this severe level of alteration in locomotion (Engel et al., 2003; Thomsen et al., 2008; Channon et al., 2009).

In the current study, raters were not part of a strong training program to reach acceptable reliability and agreement. Training increases reliability and agreement of raters (March et al., 2007; Gibbons et al., 2012; Vasseur et al., 2013). Results in the present experiment suggest that evaluation of training on raters should be performed not only for PA and $\kappa$-like statistics but also for agreement in specific levels of the scale. No training program is available for locomotion scoring such as is available for other visual scores such as body condition score (Vasseur et al., 2013) or injury scoring (Gibbons et al., 2012). In training programs, it is important to consider that the response of raters to the training may vary, with raters performing better or worse after training (Engel et al., 2003). After being trained, raters should also have periodical additional training sessions to avoid the "drift effect," which is an unconscious drift from the original definitions of the observed characteristics (Kazdin, 1977) and to ensure acceptable reliability and agreement values over time. Under practical conditions, however, periodic training sessions are not always feasible because of cost or geographical distance. Therefore, the use of experienced raters without further training together is a realistic situation that may be faced in different programs using locomotion scoring for lameness control.

The selection of the best combination of levels to produce consistent and reproducible results for locomotion scoring should be based on acceptable reliability and agreement values but also on minimizing the loss of resolution associated with merging levels. Acceptance thresholds for intrarater reliability and agreement and specific intrarater agreement for all levels in the locomotion score was met in the 4-level scale with combination $1(23) 45$, suggesting that experienced raters were able to score locomotion consistently without an excessive loss of resolution. However, moderate overall interrater reliability and agreement and specific interrater agreement for locomotion score 1(23)45 acted as a limiting factor for the selection of this combination. Overall intra- and interrater reliability and agreement and specific intra- and interrater agreement were met only in the 2-level scales (12)(345) and (123)(45). Acceptable reliability and agreement values, however, were reached at maximum loss of resolution (2-level scale).

Because 2-level scales with combinations (12)(345) and (123)(45) had acceptable reliability, agreement, and specific agreement values, the selection of one combination would depend on different factors. One factor is related to the description of the lameness status of cows. In the literature, the 2-level combination (12)(345) is the most used to classify cow as nonlame (levels 1 and 2) and lame (levels 3, 4, and 5; Winckler and Willen, 2001; Katsoulos and Christodoulopoulos, 2009; Hoffman et al., 2013). The 2-level combination (123)(45) is also commonly used to classify cows as lame (levels 4 and 5; Bicalho et al., 2007a,b; Ito et al., 2010). It is common practice to use both locomotion scores [(12)(345) and (123)(45)] to describe lameness and severe lameness (Bicalho et al., 2007b; Ito et al., 2010). Another criterion to select the best combination $[(12)(345)$ or (123)(45)] may be the capability to detect hoof lesions. Locomotion score (12)(345) presented the best sensitivity-specificity trade-off for the detection 
of painful lesions (defined as a reaction to pressure; Bicalho et al., 2007a).

A limitation of this study is the selection of arbitrary acceptance thresholds to classify reliability and agreement values as good. In this regard, $\kappa$-like statistics present a large range of acceptance thresholds from 0.4 (March et al., 2007; Burn and Weir, 2011) to 0.8 (Vasseur et al., 2013). Performing locomotion scoring under different practical conditions with an actual 4-, 3 -, or 2-level scale might result in different agreement and reliability values than those obtained in the present study. In this regard, agreement and reliability reported in the present study for locomotion scores with 4-, 3-, and 2-level scales may be used only as guidelines.

\section{CONCLUSIONS}

When locomotion scoring was performed by experienced raters without further training together, specific intra- and interrater agreement had lower values for levels 2 and 3, suggesting that experienced raters had difficulties differentiating among these 2 levels. Acceptable overall intrarater reliability and agreement and specific intrarater agreement were achieved when the 5-level scale was transformed into a 4-level scale (levels 2 and 3 merged). However, acceptable overall interrater reliability and agreement and specific interrater agreement were exceeded only when the 5-level scale was transformed into a 2-level scale when levels were merged as (12)(345) or as (123)(45). Therefore, acceptable reliability and agreement values were obtained only with an important loss of resolution of locomotion scores.

\section{ACKNOWLEDGMENTS}

This study is part of the Marie Curie Initial Training Network BioBusiness project (Marie Curie, Leuven, Belgium; FP7-PEOPLE-ITN-2008). The authors are very grateful to Jos Metz (Wageningen University, Wageningen, the Netherlands) and the reviewers for their valuable comments.

\section{REFERENCES}

Barker, Z. E., K. A. Leach, H. R. Whay, N. J. Bell, and D. C. J. Main. 2010. Assessment of lameness prevalence and associated risk factors in dairy herds in England and Wales. J. Dairy Sci. 93:932-941.

Bicalho, R. C., S. H. Cheong, G. Cramer, and C. L. Guard. 2007a. Association between a visual and an automated locomotion score in lactating Holstein cows. J. Dairy Sci. 90:3294-3300.

Bicalho, R. C., F. Vokey, H. N. Erb, and C. L. Guard. 2007b. Visual locomotion scoring in the first seventy days in milk: Impact on pregnancy and survival. J. Dairy Sci. 90:4586-4591.

Brown, L. D., T. T. Cai, and A. DasGupta. 2001. Interval estimation for a proportion. Stat. Sci. 16:101-133.
Burn, C. C., and A. A. S. Weir. 2011. Using prevalence indices to aid interpretation and comparison of agreement ratings between two or more observers. Vet. J. 188:166-170.

Channon, A. J., A. M. Walker, T. Pfau, I. M. Sheldon, and A. M. Wilson. 2009. Variability of Manson and Leaver locomotion scores assigned to dairy cows by different observers. Vet. Rec. 164:388392.

Chapinal, N., A. M. de Passillé, J. Rushen, and S. Wagner. 2010. Automated methods for detecting lameness and measuring analgesia in dairy cattle. J. Dairy Sci. 93:2007-2013.

Chapinal, N., A. M. de Passillé, D. M. Weary, M. A. G. von Keyserlingk, and J. Rushen. 2009. Using gait score, walking speed, and lying behavior to detect hoof lesions in dairy cows. J. Dairy Sci. 92:4365-4374.

Cicchetti, D. V., and A. R. Feinstein. 1990. High agreement but low kappa: II. Resolving the paradoxes. J. Clin. Epidemiol. 43:551558.

Cohen, J. 1960. A coefficient of agreement for nominal scales. Educ. Psychol. Meas. 20:37-46.

Cohen, J. 1968. Weighted kappa: Nominal scale agreement with provision for scaled disagreement or partial credit. Psychol. Bull. $70: 213-220$.

DairyCo. 2007. DairyCo Mobility Score. Vol. 2011. DairyCo, Kenilworth, UK.

Danscher, A. M., J. M. D. Enemark, E. Telezhenko, N. Capion, C. T. Ekstrom, and M. B. Thoefner. 2009. Oligofructose overload induces lameness in cattle. J. Dairy Sci. 92:607-616.

de Mol, R. M., G. André, E. J. B. Bleumer, J. T. N. van der Werf, Y. de Haas, and C. G. van Reenen. 2013. Applicability of day-to-day variation in behavior for the automated detection of lameness in dairy cows. J. Dairy Sci. 96:3703-3712.

de Vet, H. C. W., C. B. Terwee, D. L. Knol, and L. M. Bouter. 2006. When to use agreement versus reliability measures. J. Clin. Epidemiol. 59:1033-1039.

Engel, B., G. Bruin, G. Andre, and W. Buist. 2003. Assessment of observer performance in a subjective scoring system: Visual classification of the gait of cows. J. Agric. Sci. 140:317-333.

Fleiss, J. L., J. Cohen, and B. S. Everitt. 1969. Large-sample standard errors of kappa and weighted kappa. Psychol. Bull. 72:323-327.

Flower, F. C., and D. M. Weary. 2006. Effect of hoof pathologies on subjective assessments of dairy cow gait. J. Dairy Sci. 89:139-146.

Flower, F. C., and D. M. Weary. 2009. Gait assessment in dairy cattle. Animal 3:87-95.

Franzen, M. D. 2000. Reliability and Validity in Neuropsychological Assessment. Kluwer Academic/Plenum Publisher, New York, NY.

Gibbons, J., E. Vasseur, J. Rushen, and A. M. de Passillé. 2012. A training programme to ensure high repeatability of injury scoring of dairy cows. Anim. Welf. 21:379-388.

Graham, P., and B. Bull. 1998. Approximate standard errors and confidence intervals for indices of positive and negative agreement. J. Clin. Epidemiol. 51:763-771.

Hoehler, F. K. 2000. Bias and prevalence effects on kappa viewed in terms of sensitivity and specificity. J. Clin. Epidemiol. 53:499503

Hoffman, A. C., D. A. Moore, J. R. Wenz, and J. Vanegas. 2013. Comparison of modeled sampling strategies for estimation of dairy herd lameness prevalence and cow-level variables associated with lameness. J. Dairy Sci. 96:5746-5755.

Ito, K., M. A. G. von Keyserlingk, S. J. LeBlanc, and D. M. Weary. 2010. Lying behavior as an indicator of lameness in dairy cows. J. Dairy Sci. 93:3553-3560.

Katsoulos, P. D., and G. Christodoulopoulos. 2009. Prevalence of lameness and of associated claw disorders in Greek dairy cattle industry. Livest. Sci. 122:354-358.

Kazdin, A. E. 1977. Artifact, bias, and complexity of assessment: The ABCs of reliability. J. Appl. Behav. Anal. 10:141-150.

Kottner, J., L. Audigé, S. Brorson, A. Donner, B. J. Gajewski, A. Hróbjartsson, C. Roberts, M. Shoukri, and D. L. Streiner. 2011. Guidelines for reporting reliability and agreement studies (GRRAS) were proposed. J. Clin. Epidemiol. 64:96-106. 
Landis, J. R., and G. G. Koch. 1977. Measurement of observer agreement for categorical data. Biometrics 33:159-174.

Main, D. C. J., Z. E. Barker, K. A. Leach, N. J. Bell, H. R. Whay, and W. J. Browne. 2010. Sampling strategies for monitoring lameness in dairy cattle. J. Dairy Sci. 93:1970-1978.

Manson, F. J., and J. D. Leaver. 1988. The influence of dietary protein intake and of hoof trimming on lameness in dairy cattle. Anim. Prod. 47:191-199.

March, S., J. Brinkmann, and C. Winkler. 2007. Effect of training on the inter-observer reliability of lameness scoring in dairy cattle. Anim. Welf. 16:131-133.

Martin, P., and P. Bateson. 1993. Measuring Behaviour: An Introductory Guide. 2nd ed. Cambridge University Press, Cambridge, UK.

O'Callaghan, K. A., P. J. Cripps, D. Y. Downham, and R. D. Murray. 2003. Subjective and objective assessment of pain and discomfort due to lameness in dairy cattle. Anim. Welf. 12:605-610.

Rutherford, K. M. D., F. M. Langford, M. C. Jack, L. Sherwood, A. B. Lawrence, and M. J. Haskell. 2009. Lameness prevalence and risk factors in organic and non-organic dairy herds in the United Kingdom. Vet. J. 180:95-105.

Sprecher, D. J., D. E. Hostetler, and J. B. Kaneene. 1997. A lameness scoring system that uses posture and gait to predict dairy cattle reproductive performance. Theriogenology 47:1179-1187.

Thomsen, P. T., L. Munksgaard, and F. A. Togersen. 2008. Evaluation of a lameness scoring system for dairy cows. J. Dairy Sci 91:119-126.
Van Hertem, T., E. Maltz, A. Antler, C. E. B. Romanini, S. Viazzi, C. Bahr, A. Schlageter-Tello, C. Lokhorst, D. Berckmans, and I. Halachmi. 2013. Lameness detection based on multivariate continuous sensing of milk yield, rumination, and neck activity. J. Dairy Sci 96:4286-4298.

Vasseur, E., J. Gibbons, J. Rushen, and A. M. de Passillé. 2013. Development and implementation of a training program to ensure high repeatability of body condition scoring of dairy cows. J. Dairy Sci. 96:4725-4737.

Viazzi, S., C. Bahr, A. Schlageter-Tello, T. Van Hertem, C. E. B. Romanini, A. Pluk, I. Halachmi, C. Lokhorst, and D. Berckmans. 2013. Analysis of individual classification of lameness using automatic measurement of back posture in dairy cattle. J. Dairy Sci $96: 257-266$

Welfare Quality. 2009. Assessment Protocol for Cattle. Welfare Quality Consortium, Lelystad, the Netherlands.

Whay, H. 2002. Locomotion scoring and lameness detection in dairy cattle. In Pract. 24:444-449.

Winckler, C., and S. Willen. 2001. The reliability and repeatability of a lameness scoring system for use as an indicator of welfare in dairy cattle. Acta Agric. Scand. A Anim. Sci. 51:103-107 